\title{
Transmission Error of Static Characteristic for Planetary Gear Transmission in the Field of High-power Tractor
}

\author{
Xianqing Lei ${ }^{1, a^{*}}$, Zhenhua Cai ${ }^{1, b}$ and Mingzhu Zhang ${ }^{1, c}$ \\ ${ }^{1}$ Henan University of Science \& Technology Luoyang China

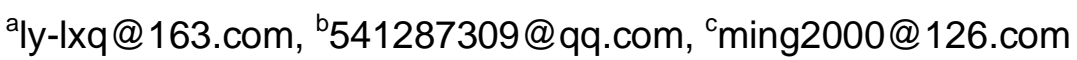

\begin{abstract}
Keywords: Tractor,Planetary gear,Static characteristic,Transmission error
Abstract. According to the structural characteristics of planetary gear transmission in the field of high-power tractor, a statics of calculative model for the load distribution of planetary gear transmission system is established. With the theory of equivalent mesh error, the main factors which affecting the transmission error of the planetary gear transmission are obtained. With the method of controlling variables, the influence law of these main factors to load sharing coefficient is researched. The most severely and acutely effects of error factors are obtained. According to the coupling formula of transmission error when the phase angle is the same, the main error factors of planetary gear transmission are coupled. Several groups of coupling schemes are put forward. Under these coupling schemes, the transmission error of planetary gear transmission is explored. The most severely and acutely factors which affecting the transmission error are obtained. According to these coupling schemes, improvement measures are put forward to reduce the transmission error. The scientific theoretical basis is provided for designing of planetary gear transmission in the high-power tractor.
\end{abstract}

\section{Introduction}

Planetary gear transmission has advantages of small volume, light weight and high bearing capacity. It is increasingly wide applicated in tractor. The accuracy and efficiency of transmission will be affected if there is a transmission error in the transmission process of planetary gear transmission.

In the planetary gear transmission, all kinds of error factors have a certain relationship between load distribution[1-2]. The load average performance of planetary gear transmission is obtained by the method of static analysis[3-6]. Some measures which improving the accuracy of plantery gear transmission are put forword by using the method of establishing the equivalent model of transmission error[7]. With the theory of equivalent mesh error, the influence of equivalent mesh error on the transmission error of planetary gear transmission is obtained[8-9].

The factor of transmission error is not just the equivalent meshing error in the process of planetary gear transmission. There are many other influence factors. There is a coupling relationship between these factors, it is less researched. According to the question of transmission error of planetary gear transmission, low-power transmission parts are researched more than high-power transmission parts. The planetary gear transmission of 300 house-power of Dongfanghong is selected as the research object. The main factors which affecting the transmission error of planetary gear transmission are explored. With the method of controlling variables, the influence law of these main factors to load sharing coefficient is researched. The most severely and acutely effects of error factors are obtained. According to the coupling formula of transmission error when the phase angle is the same, several groups of coupling schemes are put forward. According to these coupling schemes, improvement measures are put forward to reduce the transmission error.

\section{Transmission Error of Planetary Gear Transmission}

The Main Factors of Transmission Error. The transmission error of planetary gear transmission is the difference between the actual angle and the theoretical angle. The effect factors of planetary gear transmission are equivalent mesh error and manufacturing and installation errors of sun gear, planetary gear, planet carrier and annular gear. The manufacturing and installation errors are converted to the gear mesh line is equivalent mesh error[1]. The equivalent mesh error is shown in 
table 1. E and A are manufacturing and installation error. $\beta$ and $\gamma$ are the phase angle of manufacturing and installation error. $\mathrm{s}, \mathrm{c}, \mathrm{p}_{\mathrm{i}}, \mathrm{I}$ and $\mathrm{b}$ are sun gear, planet carrier, planetary gear, annular gear and bearing. $\omega$ is agular frequency. $\alpha_{w}$ and $\alpha_{n}$ are outer and inner meshing angel. $t$ is time. $\varphi_{i}$ is position angel of planetary gear.

Table 1 Equivalent mesh error

\begin{tabular}{cc}
\hline error term & equivalent mesh error \\
\hline$\left(E_{s}, \beta_{s}\right)$ & $\left.e_{E s i}=-E_{s} \sin \left[\omega_{s}-\omega_{c}\right) t+\beta_{s}+\alpha_{w}-\varphi_{i}\right]$ \\
$\left(A_{s}, \gamma_{s}\right)$ & $e_{A s i}=-A_{s} \sin \left(\omega_{c} t+\gamma_{s}+\alpha_{w}-\varphi_{i}\right)$ \\
$\left(E_{b s}, \beta_{b s}\right)$ & $\left.e_{E b s i}=-E_{b s} \sin \left[\omega_{s}-\omega_{c}\right) t+\beta_{b s}+\alpha_{w}-\varphi_{i}\right]$ \\
$\left(E_{p i}, \beta_{p i}\right)$ & $e_{E s p i}=-E_{p i} \sin \left[\left(\omega_{p}-\omega_{c}\right) t+\beta_{p i}+\alpha_{w}\right]$ \\
$\left(A_{p i}, \gamma_{p i}\right)$ & $e_{A s p i}=-A_{p i} \sin \left(\gamma_{p i}+\alpha_{w}\right)$ \\
$\left(E_{b p i}, \beta_{b p i}\right)$ & $\left.e_{E b s p i}=-E_{b p i} \sin \left[\omega_{p}-\omega_{c}\right) t+\beta_{b p i}+\alpha_{w}\right]$ \\
$\left(E_{c}, \beta_{c}\right)$ & $e_{E c s i}=E_{c} \sin \left(\beta_{c}-\varphi_{i}\right) \cos \alpha_{w}$ \\
$\left(E_{I}, \beta_{I}\right)$ & $e_{E I i}=E_{I} \sin \left(-\omega_{c} t+\beta_{I}-\alpha_{n}-\varphi_{i}\right)$ \\
$\left(A_{I}, \gamma_{I}\right)$ & $e_{A I i}=A_{I} \sin \left(-\omega_{c} t+\gamma_{I}-\alpha_{n}-\varphi_{i}\right)$ \\
$\left(E_{p i}, \beta_{p i}\right)$ & $e_{E p i I}=E_{p i} \sin \left[\left(\omega_{p}-\omega_{c}\right) t+\beta_{p i}-\alpha_{n}\right]$ \\
$\left(A_{p i}, \gamma_{p i}\right)$ & $e_{A p i I}=A_{p i} \sin \left(\gamma_{p i}-\alpha_{n}\right)$ \\
$\left(E_{b p i}, \beta_{b p i}\right)$ & $\left.e_{E b p i l}=E_{b p i} \sin \left[\omega_{p}-\omega_{c}\right) t+\beta_{b p i}-\alpha_{n}\right]$ \\
$\left(E_{c}, \beta_{c}\right)$ & $e_{E c I i}=-E_{c} \sin \left(\beta_{c}-\varphi_{i}\right) \cos \alpha_{n}$ \\
\hline
\end{tabular}

The Influence of Main Factors to Load Sharing Coefficient. The load sharing property of planetary gear transmission is reflected by load sharing coefficient. The load sharing coefficient is denoted by $\Omega$ [4]. The static load model of planetary gear is shown in figure $1 . \mathrm{K}$ is the gear mesh stiffness. $B_{i}$ is the azimuth angle of planetary gear and annular gear. $A_{i}$ is the azimuth angle of sun gear and planetary gear. $\mathrm{r}_{\mathrm{b}}$ is base radius. The machining error and installation error are $5 \mu \mathrm{m}$.

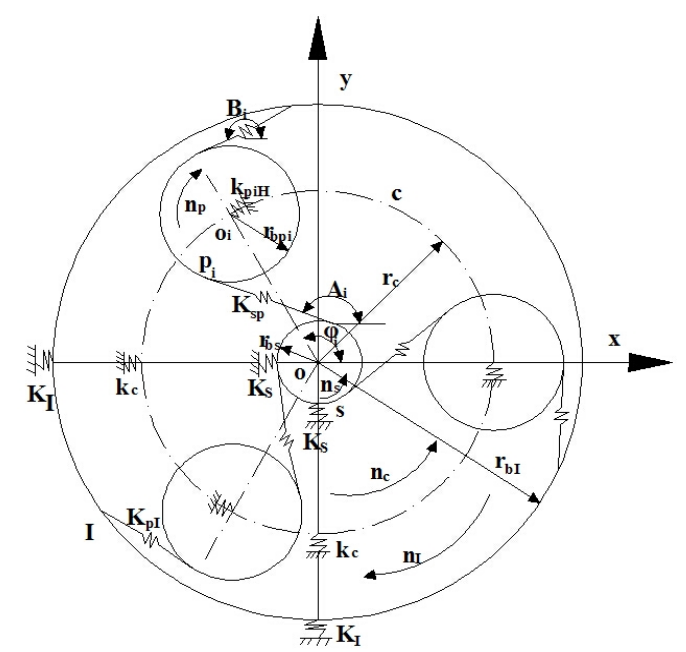

Figure 1 Statics model of planetary gear

The module is $5 \mathrm{~mm}$. The pressure angle is $20^{\circ}$. Load sharing coefficient of planetary gear is[7]:

$$
\Omega_{p i}=W_{s p i} /\left(\sum_{=1}^{n} W_{s p i} / n\right)=W_{s p i}\left(n r_{b s}\right) / T \text {. }
$$

In the formula: $W_{s p i}$ is tooth surface load of sun gear and planetary gear. $W_{P i I}$ is tooth surface load of planetary gear and annular gear. $\mathrm{T}$ is input torque. $\mathrm{p}_{\mathrm{i}}$ is the ith planetary gear. Under individual effect of factors, the change rule of load sharing coefficient is shown in figure 2. 


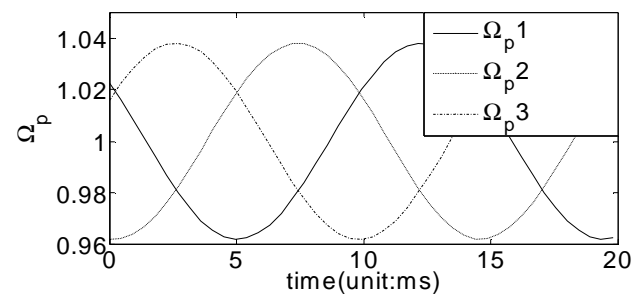

(a) Individual effect of $\mathrm{E}_{\mathrm{s}}$

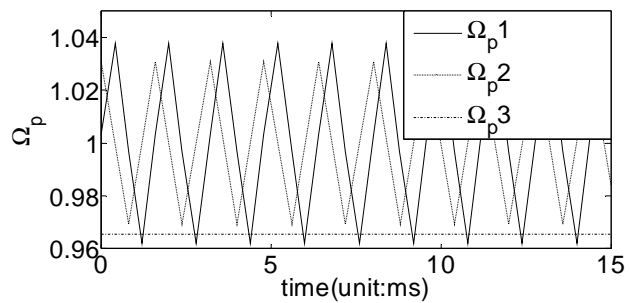

(c) Individual effect of $\mathrm{E}_{\mathrm{I}}$

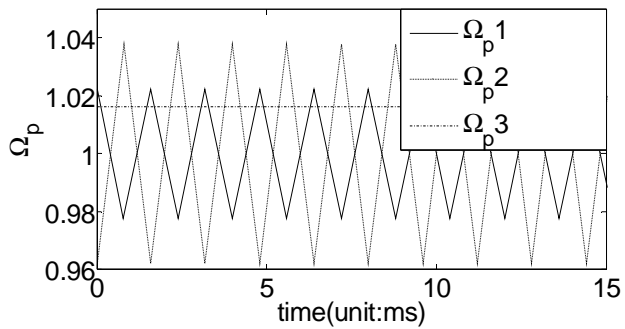

(e) Individual effect of $\mathrm{A}_{\mathrm{s}}$

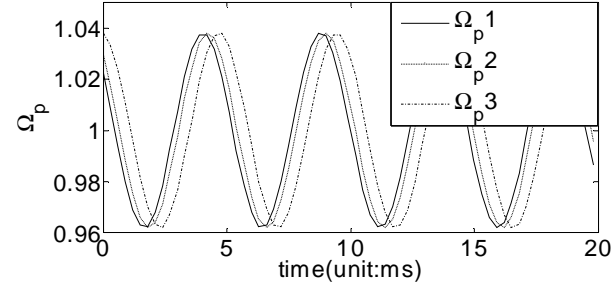

(b) Individual effect of $E_{p}$

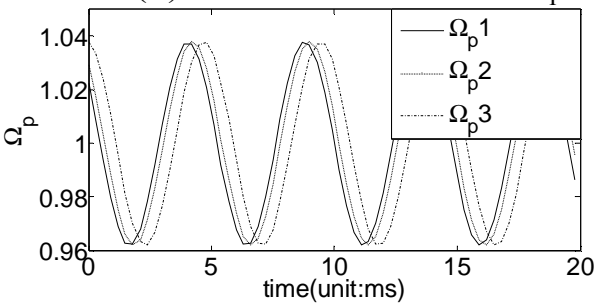

(d) Individual effect of $E_{b p}$

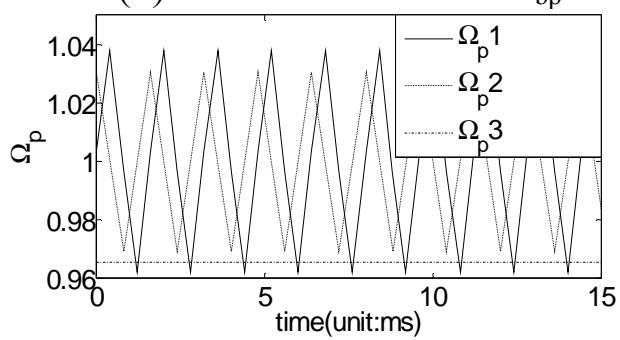

(f) Individual effect of $A_{I}$

Figure 2 The change rule of load sharing coefficient

From figure 2, the equivalent mesh error of sun gear and planetary gear have the greatest impact on load sharing coefficient. Equivalent mesh error of annular gear have the minimal impact on load sharing coefficient. With the change of time, the equivalent mesh error of planetary gear and the annular gear have the most dramatic impact on load sharing coefficient. The equivalent mesh error of sun gear have the most gentle impact on load sharing coefficient.

The coupling of Transmission Error. The sun gear input, planet carrier output, the formula of transmission error is obtained[10]:

$$
\Delta \theta_{s c}=\theta_{c}-\theta_{s} / i_{s c}=e_{w} /\left(1000 r_{b s} i_{s c}\right)-e_{n} i_{s p}^{c} /\left(1000 r_{b p} i_{s c}\right) .
$$

In the formula, $i$ is transmission ratio. $\omega_{s}$ is the angular frequency of sun gear. According to the coupling error formula, several coupling schemes of transmission error are shown in table 2.

Table 2 Coupling schemes

\begin{tabular}{cc}
\hline scheme & transmission error \\
\hline combination & $8.25^{\prime \prime}$ \\
single & $4.57^{\prime \prime}$ \\
$e_{A s p i}+e_{A p i I}$, & 0 \\
$e_{A c i}+e_{A c i}$ & $8.59^{\prime \prime}$ \\
$e_{E b s p i}+e_{E p i I}$, & $3.13^{\prime \prime}$ \\
$e_{A s i}+e_{E I i}+e_{A c i}+e_{A c i}$ & $0.55^{\prime \prime}$ \\
\hline
\end{tabular}

From the table above, some points can be obtained.

(1)When the scheme of $e_{A s p i}+e_{A p i I}$ alone, two angular frequency of error are the same. Transmission error can be directly coupled by letting $\gamma_{p}=0$ in the assembly. Transmission error before coupled is $9.14^{\prime \prime}$, the error after coupled is zero.

(2)When the scheme of $e_{A c i}+e_{A c i}^{\prime}$ alone, two angular frequency of error are the same. 
Transmission error can be directly coupled. Transmission error before coupled is 9.14", the error after coupled is $8.59^{\prime \prime}$.

(3) When the scheme of $e_{E b s p i}+e_{E p i I}$ alone, two angular frequency of error are the same. Transmission error can be directly coupled. Transmission error before coupled is $9.14^{\prime \prime}$, the error after coupled is $3.13^{\prime \prime}$.

(4)When the scheme of $e_{A s i}+e_{E I i}+e_{A c i}+e_{A c i}{ }^{\prime}$ alone, some programs can be obtained. In the assembly, the benchmark debugging on the sun gear, planetary gear, planet carrier is maked as $\beta_{I}$. Transmission error before coupled is $13.71^{\prime \prime}$, the error after coupled is $0.55^{\prime \prime}$.

\section{Conclusions}

(1)Three planetary gears are cyclical change.

(2)Equivalent mesh error of sun gear and planetary gear have the greatest impact on load sharing coefficient. Equivalent mesh error of annular gear have the minimal impact on load sharing coefficient. With the change of time, the equivalent mesh error of planetary gear and the annular gear have the most dramatic impact on load sharing coefficient. The equivalent mesh error of sun gear have the most gentle impact on load sharing coefficient.

(3)When the equivalent mesh error of sun gear alone, the load sharing coefficient of the first planetary gear and the second planetary gear have the contrary developmental trend.

(4)According to the method of coupling, the transmission error of planetary gear transmission can be reduced a lot.

\section{Acknowledgments}

The authors gratefully acknowledge National Natural Science Foundation of China (No. 51375145) for financial support of this research work.

\section{References}

[1] Hidaka Teruaki, Yamamoto Nobuyuki and Takeshi Ishida:Japan Machinery Association proceedings(Tokyo, Japan, November 8-10, 1986). Vol. 52, pp.2200-2206. (In Japanese)

[2] Hidaka Teruaki, Yamamoto Nobuyuki and Takeshi Ishida:Japan Machinery Association proceedings(Tokyo, Japan, November 5-7, 1994). Vol. 60, pp.645-653. (In Japanese)

[3] T.L. Krantz: NASA Technical Memorandum, Vol. 10(1996) No.3, pp.1-10.

[4] Ahmet: Mech Mach Theory, Vol. 29(1994) No.8, pp.1151-1161.

[5] J.G. Blanche: AEME Journal of Mechanisma, transmissions, and Automation in Design, Vol. 10(1989) No.9, pp.337-344.

[6] F.L. Litvin: Mathematical and Computer Modelling, Vol. 10(2005) No.5, pp.1063-1078.

[7] B.Li: Analyze the Method of Load Sharing Coefficient of Planetary Gear (MS., Nanjing University of Aeronautics and Astronautics, China 2005), pp.13-17. (In Chinese)

[8] F.M.Liu: Load Sharing of Multiple Planetary Gear under the Dynamic Characteristic(MS., Xiangtan University, China 2009), pp.21-25. (In Chinese)

[9] C.B.Wang:Journal of Chongqing University, Vol. 9(2012) No.4, pp.66-70.(In Chinese)

[10]C.B.Wang:Transactions of the Chinese Society for Agricultural Machinery, Vol. 8(2013) No.49, pp.1000-1002.(In Chinese) 